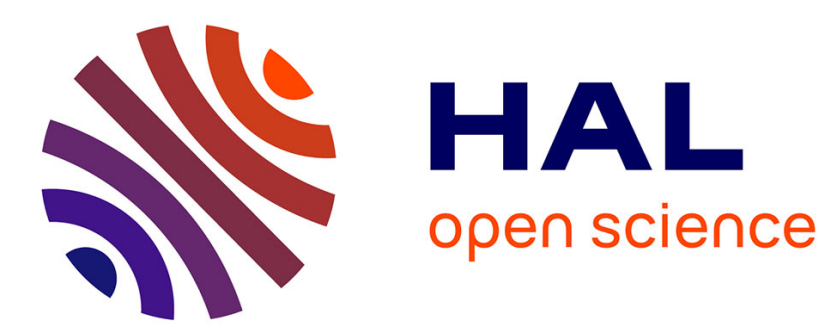

\title{
On some inequalities of Bourgain, Brezis, Maz'ya, and Shaposhnikova related to $L^{1}$ vector fields
}

Petru Mironescu

\section{To cite this version:}

Petru Mironescu. On some inequalities of Bourgain, Brezis, Maz'ya, and Shaposhnikova related to $L^{1}$ vector fields. Comptes rendus de l'Académie des sciences. Série I, Mathématique, 2010, 348 (9-10), pp.513-515. 10.1016/j.crma.2010.03.019 . hal-00747678

\section{HAL Id: hal-00747678 \\ https://hal.science/hal-00747678}

Submitted on 31 Oct 2012

HAL is a multi-disciplinary open access archive for the deposit and dissemination of scientific research documents, whether they are published or not. The documents may come from teaching and research institutions in France or abroad, or from public or private research centers.
L'archive ouverte pluridisciplinaire HAL, est destinée au dépôt et à la diffusion de documents scientifiques de niveau recherche, publiés ou non, émanant des établissements d'enseignement et de recherche français ou étrangers, des laboratoires publics ou privés. 


\title{
On some inequalities of Bourgain, Brezis, Maz'ya, and Shaposhnikova related to $L^{1}$ vector fields
}

\author{
Petru Mironescu *
}

March 23rd, 2010

\begin{abstract}
Bourgain and Brezis established, for maps $f \in L^{n}\left(\mathbb{T}^{n}\right)$ with zero average, the existence of a solution $\vec{Y} \in W^{1, n} \cap L^{\infty}$ of (1) $\operatorname{div} \vec{Y}=f$. Maz'ya proved that if, in addition, $f \in H^{n / 2-1}\left(\mathbb{T}^{n}\right)$, then (1) can be solved in $H^{n / 2} \cap L^{\infty}$. Their arguments are quite different. We present an elementary property of fundamental solutions of the biharmonic operator in two dimensions. This property unifies, in two dimensions, the two approaches, and implies another (apparently unrelated) estimate of Maz'ya and Shaposhnikova. We discuss higher dimensional analogs of the above results.
\end{abstract}

\section{Résumé}

Sur certaines inégalités de Bourgain, Brezis, Maz'ya et Shaposhnikova concernant les champs de vecteurs dans $L^{1}$. Bourgain and Brezis ont montré que, si $f \in L^{n}\left(\mathbb{T}^{n}\right)$ est de moyenne nulle, alors (1) $\operatorname{div} \vec{Y}=f$ a une solution $\vec{Y} \in W^{1, n} \cap C^{0}$. Maz'ya a prouvé que si, de plus, on a $f \in H^{n / 2-1}\left(\mathbb{T}^{n}\right)$, alors il existe une solution de (1) dans $H^{n / 2} \cap L^{\infty}$. Les deux preuves sont distinctes. Dans cette note, nous présentons une propriété élémentaire des solutions fondamentales de l'opérateur biharmonique en dimension deux. Cette propriété unifie, en dimension deux, les approches de Bourgain-Brezis et Maz'ya, et implique une autre estimation de Maz'ya et Shaposhnikova (apparemment non liée aux précédentes). Nous discutons des variantes de ces résultats en dimension supérieure.

In their pioneering work [1], Bourgain and Brezis proved that, when a map $f \in L^{n}\left(\mathbb{T}^{n}\right)$ has zero average,

$$
\operatorname{div} \vec{Y}=f
$$

has a solution $\vec{Y} \in W^{1, n} \cap C^{0}$. By duality, this result is trivially equivalent to the estimate

$$
\|u\|_{L^{n /(n-1)}} \lesssim\|\nabla u\|_{W^{-1, n^{\prime}+L^{1}}}, \quad \forall u \in L^{n}\left(\mathbb{T}^{n}\right) \text { such that } \int_{\mathbb{T}^{n}} u=0
$$

The proof of (1) is very elaborate (the construction of $\vec{Y}$ is explicit and based on a nonlinear mechanism). So far, there is no straightforward argument yielding (2) when $n \geq 3$. However, when $n=2$, Bourgain and Brezis [1] present a direct proof of (2) which relies on Fourier series, and more specifically on the fact that

$$
\sum_{m \in \mathbb{Z}^{2} \backslash\{0\}} \frac{m_{1} m_{2}}{\left(m_{1}^{2}+m_{2}^{2}\right)^{2}} e^{\imath m \cdot x} \in L^{\infty} \quad \text { and } \sum_{m \in \mathbb{Z}^{2} \backslash\{0\}} \frac{m_{1}^{2}-m_{2}^{2}}{\left(m_{1}^{2}+m_{2}^{2}\right)^{2}} e^{\imath m \cdot x} \in L^{\infty} .
$$

${ }^{*}$ Université de Lyon, Université Lyon1, CNRS, UMR 5208 Institut Camille Jordan, Bâtiment du Doyen Jean Braconnier, 43, blvd du 11 novembre 1918, F - 69200 Villeurbanne Cedex, France. Email address: mironescu@math.univlyon1.fr 
Assertion (1) is equivalent to the fact that for every vector field $\vec{X} \in W^{1, n}\left(\mathbb{T}^{n}\right)$ there is some $\vec{Y} \in$ $W^{1, n} \cap C^{0}$ such that $\vec{X}=\vec{Y}+\vec{Z}$, where $\operatorname{div} \vec{Z}=0$. For $n \geq 3$, a more involved version of this this result has been established by Bourgain and Brezis [2], [3]: in the previous decomposition, one may pick $\vec{Z}$ such that curl $\vec{Z}=0$. This implies new regularity results for the Hodge decomposition [2], [3]. For example, when $n=3$, Bourgain and Brezis [3] prove, for vectors fields $\vec{f} \in L^{3}\left(\mathbb{T}^{3}\right)$ such that div $\vec{f}=0$ and $\int_{\mathbb{T}^{3}} \vec{f}=0$, the existence of a $\vec{Y} \in W^{1,3} \cap C^{0}\left(\mathbb{T}^{3}\right)$ such that

$$
\operatorname{curl} \vec{Y}=\vec{f}
$$

Maz'ya [4] studied the solvability of (1) when $f \in H^{n / 2-1}\left(\mathbb{T}^{n}\right)$ has zero average. The main result there is the existence of a solution $\vec{Y} \in H^{n / 2} \cap L^{\infty}$ of (1). The proof of Maz'ya [4] is by duality, based on the estimate

$$
\|u\|_{H^{1-n / 2}} \lesssim\|\nabla u\|_{H^{-n / 2}+L^{1}}, \quad \forall u \in H^{1-n / 2}\left(\mathbb{T}^{n}\right) \operatorname{such} \int_{\mathbb{T}^{n}} u=0 .
$$

Actually, [4] contains a version of (5) in $\mathbb{R}^{n}$ instead of $\mathbb{T}^{n}$ and with sharp constants. The proof of (5) is based on explicit formulae for the Fourier transform of singular integral operators, in the spirit of Stein, Weiss [7], Chapter IV, Theorem 4.5, p. 164. In dimension two, (5) is the same as (2) and provides a third argument leading to the solvability of (1) in $H^{1} \cap C^{0}\left(\mathbb{T}^{2}\right)$.

In a different direction, Maz'ya and Shaposhnikova [6] proved the following estimates: for $u \in$ $C^{\infty}\left(\mathbb{T}^{n}\right)$, one has

$$
\left|\int_{\mathbb{T}^{n}} \partial_{1} u \partial_{2} u\right|+\left|\int_{\mathbb{T}^{n}}\left(\left(\partial_{1} u\right)^{2}-\left(\partial_{2} u\right)^{2}\right)\right| \lesssim\left(\int\left|(-\Delta)^{n / 4+1 / 2} u\right|\right)^{2} .
$$

Their approach is again based on Fourier transform formulae for singular integral operators, in the spirit of the proofs of (5) in [4] and of the $H^{3 / 2}$-regularity result in [5], and apparently unrelated to the proof of $(2)$ via $(3)$ in $[1]$.

Our first contribution is the following: we revisit and connect, in two dimensions, (3) and (6) using a partial differential equations viewpoint. More specifically, our starting point is the following

Proposition 1 In $\mathbb{R}^{2}$, the operator $\Delta^{2}$ has a fundamental solution $F$ such that $\partial_{1} \partial_{2} F, \partial_{1}^{2} F-\partial_{2}^{2} F \in L^{\infty}$.

Proof. Let $F(x)=\frac{1}{8 \pi}|x|^{2} \ln |x|-\frac{1}{16 \pi}|x|^{2}$. One checks easily that $\partial_{j}^{2} F=\frac{1}{4 \pi} \ln |x|+\frac{1}{4 \pi} \frac{x_{j}^{2}}{|x|^{2}}, \partial_{1} \partial_{2} F=$ $\frac{1}{4 \pi} \frac{x_{1} x_{2}}{|x|^{2}}$. In particular, $\Delta F=\frac{1}{2 \pi} \ln |x|$, so that $\Delta^{2} F=\delta$, while $\partial_{1} \partial_{2} F, \partial_{1}^{2} F-\partial_{2}^{2} F \in L^{\infty}$.

Corollary 2 Let $G$ be the (unique modulo constants) solution of $\Delta^{2} G=\delta-(1 / 2 \pi)^{2}$ on $\mathbb{T}^{2}$. Then $\partial_{1} \partial_{2} G$ and $\partial_{1}^{2} G-\partial_{2}^{2} G$ belong to $L^{\infty}$.

Equivalently, (3) holds.

Proof. Let $\varphi \in C_{c}^{\infty}(B(0,1 / 2))$ with $\varphi=1$ in $B(0,1 / 4)$. Then $H=\varphi F$ may be identified with a map on $\mathbb{T}^{2}$. Since $\Delta^{2}(G-H) \in C^{\infty}$, we have $G-H \in C^{\infty}$. We conclude via $\partial_{1} \partial_{2} H, \partial_{1}^{2} H-\partial_{2}^{2} H \in L^{\infty}$.

Noting that, up to a constant, we have $G=\sum_{m \in \mathbb{Z}^{2} \backslash\{0\}} \frac{1}{\left(m_{1}^{2}+m_{2}^{2}\right)^{2}} e^{\imath m \cdot x}$, we find that Corollary 2 is equivalent to (3). 
Remark 1 Corollary 2 implies (6) when $n=2$. Here is the proof. We treat, e. g., the first integral in (6). We have

We deduce that

$$
\int \partial_{1} u \partial_{2} u=\int\left[\left(\Delta^{2} G\right) * \partial_{1} u\right] \partial_{2} u=-\int\left[\left(\partial_{1} \partial_{2} G\right) *(\Delta u)\right](\Delta u) .
$$

$$
\left|\int \partial_{1} u \partial_{2} u\right| \leq\left\|\left(\partial_{1} \partial_{2} G\right) *(\Delta u)\right\|_{L^{\infty}}\|\Delta u\|_{L^{1}} \leq\left\|\partial_{1} \partial_{2} G\right\|_{L^{\infty}}\|\Delta u\|_{L^{1}}\|\Delta u\|_{L^{1}} \lesssim\|\Delta u\|_{L^{1}}^{2}
$$

Next we discuss the higher dimensional analogs of Proposition 1 and Corollary 2, as well as their connection to (5) and (6).

Proposition 3 In $\mathbb{R}^{n}$, the operator $(-\Delta)^{n / 2+1}$ has a fundamental solution $F$ such that $\partial_{1} \partial_{2} F \in L^{\infty}$ and $\partial_{1}^{2} F-\partial_{2}^{2} F \in L^{\infty}$.

Here, when $n$ is odd, a fundamental solution $F$ is a temperate solution of $(-\Delta)^{n / 2+1 / 2} F=\mathscr{F}^{-1}\left((2 \pi|\xi|)^{-1}\right)$.

Proof. One may check that, with $\alpha_{n}:=\frac{1}{2^{n+1} \pi^{n / 2} \Gamma(n / 2+1)}$, the map $F(x):=\alpha_{n}\left\{|x|^{2} \ln |x|-|x|^{2} / 2\right\}$ is a fundamental solution. In addition, we have $\partial_{1} \partial_{2} F=2 \alpha_{n} x_{1} x_{2}|x|^{-2}$ and $\partial_{1}^{2} F-\partial_{2}^{2} F=2 \alpha_{n}\left(x_{1}^{2}-x_{2}^{2}\right)|x|^{-2}$.

The analogs of Corollary 2 and formula (3) are given by

Proposition 4 Let $G$ be the (unique up to constants) solution of $(-\Delta)^{n / 2+1} G=\delta-(1 / 2 \pi)^{n}$ on $\mathbb{T}^{n}$. Then $\partial_{1} \partial_{2} G$ and $\partial_{1}^{2} G-\partial_{2}^{2} G$ belong to $L^{\infty}$. Equivalently,

$$
\sum_{m \in \mathbb{Z}^{n} \backslash\{0\}} \frac{m_{1} m_{2}}{|m|^{n+2}} e^{\imath m \cdot x} \in L^{\infty} \quad \text { and } \sum_{m \in \mathbb{Z}^{n} \backslash\{0\}} \frac{m_{1}^{2}-m_{2}^{2}}{|m|^{n+2}} e^{\imath m \cdot x} \in L^{\infty} .
$$

Sketch of proof. When $n$ is even, $(-\Delta)^{n / 2+1}$ is a local operator, so that we may repeat the proof of Corollary 2. When $n$ is odd, we mimic the proof of (3) in [1], p. 405-406.

Remark 2 In the same way that Corollary 2 implies (6) when $n=2$, Proposition 4 implies (6) for all $n$.

Remark 3 One can recover estimate (5) of Maz'ya by combining (6) to some arguments used by Bourgain and Brezis [1] in the proof of (2). The starting point of the proof is the following estimate, reminiscent of [1], p. 404, and valid when u has zero average:

$$
\|u\|_{H^{1-n / 2}}^{2} \sim \sum_{j<k}\left(\left\|\partial_{j} \partial_{k}(-\Delta)^{-n / 4-1 / 2} u\right\|_{L^{2}}^{2}+\left\|\left(\partial_{j}^{2}-\partial_{k}^{2}\right)(-\Delta)^{-n / 4-1 / 2} u\right\|_{L^{2}}^{2}\right) .
$$

Let $\nabla u=\vec{U}+\vec{V}, \vec{U} \in H^{-n / 2}, \vec{V} \in L^{1}$. Inspired by [1], p. 403-405, we treat, e. g., the first term in (8) for $j=1, k=2$ using the identity

$$
\begin{aligned}
\left\|\partial_{1} \partial_{2}(-\Delta)^{-n / 4-1 / 2} u\right\|_{L^{2}}^{2}=\int\{ & {\left[\partial_{1} \partial_{2}(-\Delta)^{-n / 4-1 / 2} u\right]\left[(-\Delta)^{-n / 4-1 / 2}\left(\partial_{1} U_{2}+\partial_{2} U_{1}\right)\right] } \\
& -\left[\partial_{1}(-\Delta)^{-n / 4-1 / 2} U_{2}\right]\left[\partial_{2}\left[(-\Delta)^{-n / 4-1 / 2} U_{1}\right]\right. \\
+ & {\left.\left[\partial_{1}(-\Delta)^{-n / 4-1 / 2} V_{2}\right]\left[\partial_{2}(-\Delta)^{-n / 4-1 / 2} V_{1}\right]\right\} }
\end{aligned}
$$

Using standard elliptic estimates for the first two integrals on the right-hand side of (9) and Proposition 4 for the last integral, we find that

$$
\left\|\partial_{1} \partial_{2}(-\Delta)^{-n / 4-1 / 2} u\right\|_{L^{2}}^{2} \lesssim\left\|\partial_{1} \partial_{2}(-\Delta)^{-n / 4-1 / 2} u\right\|_{L^{2}}\|U\|_{H^{-n / 2}}+\|U\|_{H^{-n / 2}}^{2}+\|V\|_{L^{1}}^{2}
$$

i. e., $\|u\|_{H^{1-n / 2}} \lesssim\|U\|_{H^{-n / 2}}+\|V\|_{L^{1}}$. 


\section{Acknowledgement}

The author warmly thanks Haïm Brezis for useful discussions.

\section{References}

[1] J. Bourgain, H. Brezis, On the equation $\operatorname{div} Y=f$ and application to control of phases, J. Amer. Math. Soc. 16 (2003), 393-426.

[2] J. Bourgain, H. Brezis, New estimates for the Laplacian, the div-curl, and related Hodge systems, C. R. Acad. Sci. Paris 338 (2004), 539-543. 393-426.

[3] J. Bourgain, H. Brezis, New estimates for elliptic equations and Hodge type systems, J. Eur. Math. Soc. 9 (2007), 277-315.

[4] V. Maz'ya, Bourgain-Brezis type inequality with explicit constants, in: L. De Carli, M. Milman (Eds.), Interpolation theory and applications, Contemp. Math., 445, AMS Providence, RI, 2007, $247-252$.

[5] V. Maz'ya, Estimates for differential operators of vector analysis involving $L^{1}$-norm, J. Eur. Math. Soc. 12 (2010), 221-240.

[6] V. Maz'ya, T. Shaposhnikova, A collection of sharp dilation invariant integral inequalities for differentiable functions, in: V. Maz'ya (Ed.), Sobolev spaces in mathematics I, Int. Math. Ser. (N. Y.) 8, Springer, New York, 2009, 223-247.

[7] E. Stein, G. Weiss, Introduction to Fourier Analysis on Euclidean Spaces, Princeton University Press, Princeton, 1971. 